\title{
INSTITUTION
}

\section{Un cancérologue indépendant à la tête de l'Afssaps "Priorité au progrès, à la transparence et à la communication »}

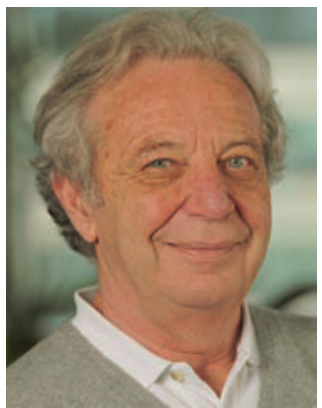

Pr Dominique Maraninchi AFSSAPS - 143/147, bd Anatole France 93285 Saint Denis Cedex

A près cinq années passées à la tête du conseil d'administration de l'INCa, le Pr Dominique Maraninchi, cancérologue marseillais de 61 ans, a été nommé directeur général de I'Agence française de sécurité sanitaire des produits de santé (Afssaps) le 22 février dernier. En plein scandale de l'antidiabétique Médiator, son absence de lien financier avec l'industrie pharmaceutique depuis 2002 aurait pesé dans la balance de sa nomination. Ainsi, le ministre de la Santé, Xavier Bertrand, a-til souligné qu'il disposait de « l'autorité et la légitimité » nécessaires pour relever le " challenge » de la remise en ordre de I'Afssaps lors du lancement des Assises du médicament en février dernier.

Quels axes souhaitez-vous donner à votre direction de l'Afssaps?

Cela peut se résumer en trois grandes notions : favoriser le progrès thérapeutique, instaurer une culture de transparence et renforcer la communication auprès des patients, des professionnels, et des industriels. Premièrement donc, un accès plus large à I'innovation et aux médicaments. Cela passe par un soutien à la recherche clinique et aux maladies orphelines. Nous souhaitons encourager les progrès. L'Afssaps a vocation dorénavant à développer et à suivre les médicaments tout au long de leur vie. Nous ne pouvons nous contenter de donner des AMM sans, par la suite, suivre le devenir des médicaments, la découverte de nouveaux effets secondaires ou de nouvelles indications, voire restreindre l'usage des molécules aux seuls patients qui en tirent des bénéfices. S'il y a un doute sur un médicament, c'est aux patients avant tout que nous devons penser. Nous devons être capables d'ajuster le tir régulièrement et d'être les plus réactifs possible pour étendre ou restreindre l'usage d'un médicament.

En termes de transparence, et notamment d'indépendance des experts, qu'est-ce que cela signifie ?

Il est indispensable que notre expertise soit indépendante et transparente. Cela signifie notamment que tous les dossiers d'instruction, tous les avis que nous rendons et les décisions qui sont prises, doivent être accessibles à tous. L'Agence doit instruire les dossiers en interne avec les compétences dont elle dispose, c'est pour cette raison qu'elles doivent absolument être développées. Nous consulterons des experts extérieurs uniquement sur des questions précises. Ces experts, dont certains pourront même faire partie d'associations de patients par exemple, devront évidemment déclarer leurs liens d'intérêt avec l'industrie pharmaceutique. II n'en reste pas moins que ces experts ne sont pas décisionnaires, ils remettent des avis au Directeur général de l'Afssaps, qui prend des décisions. Cette notion de transparence s'applique aussi à la communication envers tous les publics. Nous devons informer les patients, les prescripteurs et les fabricants des dossiers que nous instruisons.

Nous apprenons que le Pr Maraninchi vient d'être promu au grade d'officier de la Légion d'honneur le 14 juillet. Toutes nos félicitations au récipiendaire!

Synthèse du rapport de l'IGAS sur la pharmacovigilance et gouvernance de la chaîne du médicament, publiée en juin 2011 : www.igas.gouv.fr/IMG/pdf/Synthese_RM2011-103P_pharmacovigilance.pdf
Il est important d'informer sans drame car le médicament est un progrès.

De nouvelles mesures ont été annoncées en termes de pharmacovigilance, pourquoi une telle évolution?

Désormais les patients eux-mêmes, les médecins traitants et les pharmaciens d'officine pourront nous faire part, via un formulaire sur le site internet de l'Afssaps, de la survenue d'effets indésirables inattendus. En effet, il n'est pas sûr que les patients euxmêmes ne soient pas pertinents sur ce thème. J'ai moi-même écrit des articles qui relataient que les patients observent bien souvent des effets secondaires différents que ceux rapportés par les médecins. Parfois moins graves, mais ce n'est pas pour autant qu'ils sont insignifiants en termes de répercussions sur la qualité de vie des malades. Ces effets-là, qui peuvent être mineurs mais concernés un très grand nombre de patients, doivent être portés à la connaissance des prescripteurs. Cette nouvelle disposition nous oblige aussi à être en lien direct avec les malades eux-mêmes. Je suis très sensible à la remontée d'information, notamment des signaux faibles.

Plusieurs cancérologues viennent d'être nommés à la tête d'instances importantes, qu'en pensez-vous ?

Qu'un cancérologue, le Pr Josy Rieffers, prenne la direction de la FFCLCC quoi de plus naturel! Mais pour le reste, le Pr Gérard Tobelem à l'Établissement Français du Sang et le Pr Jean-Luc Harousseau à la Haute Autorité de Santé, je préfère voir cela comme un hasard. On ne peut négliger cependant le fait que l'hématologie est une discipline où la recherche et la médecine ont toujours été en étroite connexion. C'est un domaine qui par ailleurs a connu beaucoup d'évolutions, voire de révolutions, ces dernières années. Les personnes qui en sont issues font preuve d'un certain pragmatisme et d'une double lecture. Mais ce n'est pas propre à cette unique discipline.

Propos recueillis par Émilie Gillet 